\title{
SIMULTANEOUS DIAGNOSIS OF GRAVES HYPERTHYROIDISM AND ADRENAL INSUFFICIENCY
}

I Kostoglou-Athanassiou, E Kostakis, A Dermentzoglou, E Xanthakou

Department of Endocrinology, Red Cross Hospital, Athens, Greece

Endocrinologist, Sparti

\section{OBJECTIVES}

\begin{abstract}
Patients with autoimmune diseases are known to develop other autoimmune conditions. Adrenal insufficiency of autoimmune etiology is known to coexist with autoimmune diabetes mellitus, Hashimoto's thyroiditis and vitiligo. However, Graves' disease in the context of adrenal insufficiency of autoimmune etiology is rare.
\end{abstract}

The aim was to describe the case of a patient who presented with fatique and was diagnosed to have hyperthyroidism and adrenal insufficiency of autoimmune etiology.

\section{CASE REPORT}

A patient, male aged 25 years presented with fatique, tachycardia and weight loss. He was found to have high free T4 levels and TSH $0.001 \mathrm{mU} / \mathrm{L}$. Antithyroid drugs were administered. However, the patient complained of continuing fatique and weight loss. He had dark colored skin. However, when his photos were compared to older ones it appeared that his skin had a darker color. Morning cortisol and ACTH were measured. Cortisol was found to be very low and ACTH high. Cortisol was administered along with antithyroid medications and the patient improved. TSH receptor antibodies were positive and anti-adrenal antibodies were also positive. On clinical examination as well on ultrasonography the patient had no evidence of Graves' ophthalmopathy.

\section{CONCLUSIONS}

Autoimmune diseases may coexist with other autoimmune conditions, which develop usually sequentially. However, even in young patients, the simultaneous development of more than one endocrine autoimmune conditions cannot be excluded, and it should be included in the differential diagnosis, especially if symptoms persist. 